\title{
Hypoxia induces PGC-1 $\alpha$ expression and mitochondrial biogenesis in the myocardium of TOF patients
}

\author{
Lingyun Zhu ${ }^{1, *}$, Qiang Wang ${ }^{2,}$, Lin Zhang ${ }^{1, *}$, Zhixiang Fang ${ }^{1}$, Fang Zhao ${ }^{3}$, Zhiyuan Lv $^{1}$, Zuguang Gu ${ }^{1}$, \\ Junfeng Zhang ${ }^{1}$, Jin Wang ${ }^{1}$, Ke Zen ${ }^{1}$, Yang Xiang ${ }^{1}$, Dongjin Wang ${ }^{2}$, Chen-Yu Zhang ${ }^{1}$ \\ ${ }^{I}$ Jiangsu Diabetes Center, State Key Laboratory of Pharmaceutical Biotechnology, School of Life Sciences, Nanjing University, \\ Nanjing 210093, China; ${ }^{2}$ Department of Thoracic Surgery, The Affiliated Drum Tower Hospital of Nanjing University Medical \\ School, Nanjing 210008, China, ${ }^{3}$ Department of Physiology, University of Toronto, Toronto, Ontario M5S 1A8, Canada
}

PGC-1 $\alpha$, a potent transcriptional coactivator, is the major regulator of mitochondrial biogenesis and activity in the cardiac muscle. The dysregulation of PGC-1 $\alpha$ and its target genes has been reported to be associated with congenital and acquired heart diseases. By examining myocardium samples from patients with Tetralogy of Fallot, we show here that PGC-1 $\alpha$ expression levels are markedly increased in patients compared with healthy controls and positively correlated with the severity of cyanosis. Furthermore, hypoxia significantly induced the expression of PGC-1 $\alpha$ and mitochondrial biogenesis in cultured cardiac myocytes. Mechanistic studies suggest that hypoxia-induced PGC-1 $\alpha$ expression is regulated through the AMPK signaling pathway. Together, our data indicate that hypoxia can stimulate the expression of PGC-1 $\alpha$ and mitochondrial biogenesis in the cardiac myocytes, and this process might provide a potential adaptive mechanism for cardiac myocytes to increase ATP output and minimize hypoxic damage to the heart. Keywords: PGC-1 $\alpha$, mitochondria, hypoxia, myocardium, tetralogy of Fallot Cell Research (2010) 20:676-687. doi:10.1038/cr.2010.46; published online 6 April 2010

\section{Introduction}

Tetralogy of Fallot (TOF) is one of the most common types of cyanotic heart lesions and occurs in $8 \%$ to $9.7 \%$ of children with congenital heart disease $[1,2]$. TOF is a conoventricular abnormality that consists of four anatomical malformations: (1) a ventricular septal defect; (2) a pulmonary arterial stenosis; (3) an aortic valve that overrides the interventricular septum; and (4) hypertrophy of the right ventricle, which may not occur until the neonatal period $[3,4]$. TOF patients suffer from systemic chronic hypoxia and sustained pressure overload of the right ventricle. Survival is dependent on many adaptive mechanisms, including altered expression of protective angiogenic, metabolic and antioxidant genes in the

\footnotetext{
*These three authors contributed equally to this work. Correspondence: Chen-Yu Zhang ${ }^{\mathrm{a}}$, Dongjin Wang ${ }^{\mathrm{b}}$, Yang Xiang

a'E-mail: cyzhang@nju.edu.cn; Fax: +86-25-83686234

bE-mail: gldjw@163.com; Fax: +86-25-83105117

EE-mail: xiangy@nju.edu.cn; Fax:+86-25-83685616

Received 28 August 2009; revised 22 December 2009; accepted 7 February 2010; published online 6 April 2010
}

myocardium, which improve $\mathrm{O}_{2}$ delivery and energy production and minimize reoxygenation injury [5-7]. TOF patients usually undergo surgery at a young age to repair their malfunctions; however, delayed treatment of TOF is associated with maladaptive changes that result in decreased myocardial performance $[6,8]$.

The importance of mitochondria in energy production of cardiac muscles has been well documented in the last two decades. A large body of work has been established to suggest that congenital and acquired heart diseases are associated with mitochondrial abnormalities [9-11]. Reduced mitochondrial enzymatic activities $[12,13]$ and mitochondrial abnormalities $[14,15]$ have been reportedly found in the hearts of TOF patients. PGC- $1 \alpha$, a potent transcriptional coactivator of many nuclear receptors and transcription factors, serves as a major regulator of mitochondrial biogenesis and activity [16-19]. PGC-1 $\alpha$ regulates the expression of many genes that code for mitochondrial proteins. It is abundantly expressed in heart tissue and has been demonstrated to have a critical role in the expression of genes involved in the mitochondrial ATP-generating pathway in the heart, working through a panel of molecules including PPARs, estrogen-related 
receptors, nuclear respiratory factors (NRFs), and other factors, such as muscle enhancer factor-2 [20-22]. Furthermore, it has been shown that forced expression of PGC- $1 \alpha$ in mutant cardiac myocytes prevents the apoptotic phenotype of these cells [23].

Given the importance of PGC- $1 \alpha$ in heart energy metabolism, PGC- $1 \alpha$ expression in cardiac muscle is tightly modulated [20]. Expression of PGC-1 $\alpha$ is induced perinatally and gradually increases to a high level in the adult heart, accompanying physiological heart growth $[21,24]$. Pathological cardiac hypertrophy, induced by aortic banding in the mouse models of chronic pressure overload, results in a decrease in myocardial expression of both PGC-1 $\alpha[25,26]$ and its target genes that are involved in fatty acid oxidation and oxidative phosphorylation $[21,25]$. These results indicate that PGC- $1 \alpha$ expression is highly relevant to heart development and heart disease.

Because the two major risk factors of TOF patients are systemic chronic hypoxia and sustained pressure overload to the right ventricle, it is important to explore the PGC- $1 \alpha$ expression level in the right ventricle of TOF patients. In this study, we show that both PGC- $1 \alpha$ expression and mitochondrial number are significantly increased in the myocardium of TOF patients. Furthermore, PGC-1 $\alpha$ expression is increased in primary myocardial cells and myocardial cell lines cultured under hypoxic conditions, and this is accompanied by increased mitochondrial biogenesis. Mechanistic studies suggest that hypoxia-induced PGC- $1 \alpha$ expression is regulated through the AMPK signaling pathway. Thus, our results suggest that hypoxia induces the expression of PGC- $1 \alpha$ and mitochondrial biogenesis through the AMPK signaling pathway.

\section{Results}

\section{Patient characteristics}

With a total of 29 patients, 17 TOF and 12 normal subjects were studied (Table 1). TOF patients had significantly lower $\mathrm{O}_{2}$ saturation in their blood $(86 \% \pm 2 \%$ versus $98 \% \pm 2 \% ; P<0.001)$, higher mean preoperative hematocrit $(51 \% \pm 2 \%$ versus $35 \% \pm 1 \% ; P<0.001)$ and higher hemoglobin concentrations $(170 \pm 7$ versus $118 \pm$ $5 \mathrm{~g} / 1 ; P<0.001)$ when compared with the normal group. These data indicate that the TOF patients suffered from severe systemic hypoxia.

Increased mitochondrial number in the heart tissue of TOF patients

TOF is a complicated heart disease with abnormal energy metabolism caused by chronic hypoxia and sustained pressure overload. Given the vital role of mitochondria in energy metabolism, we examined the mitochondrial ultra-structure in the myocardial muscle tissue of TOF patients using transmission electron microscopy. Mitochondrial sections of the TOF myocardium exhibited vacuolar degeneration and breakage of the mitochondrial cristae. Strikingly, an increased number of mitochondria was observed in the TOF myocardium compared with the control (Figure 1A). Quantification of the mitochondria volume showed that there was a 1.6-fold increase in the myocardium of TOF patients compared with that of the control group (Figure $1 \mathrm{~B}, P<0.05$ ). The measurement of mitochondrial DNA content by real-time PCR further confirmed the increased mitochondrial biogenesis in the myocardium of TOF patients. As shown in Figure $1 \mathrm{C}$, the relative median mtDNA copy number was 1.44-fold higher in the myocardium of TOF patients than in control subjects $(P<0.05)$.

$P G C-1 \alpha$ expression is increased in the myocardial muscle of TOF patients

PGC- $1 \alpha$ is a key regulator of mitochondrial biogenesis and activity. The expression of PGC- $1 \alpha$ has been found to be altered in the myocardium of subjects with various heart diseases $[21,25,26]$. Given the mitochondrial abnormality in the cardiac muscles of TOF patients, we examined the PGC- $1 \alpha$ expression levels in the myocardial muscles of the 17 TOF patients and 12 control subjects. We found that the PGC- $1 \alpha$ mRNA level in the myocardial muscles from TOF patients was approximately 1.6fold higher than that of control subjects (Figure 2A). Interestingly, the PGC-1 $\alpha$ protein level was also found to be significantly increased in these samples (Figure 2B). The specificity of the PGC- $1 \alpha$ antibody used in this study is shown in Supplementary information, Figure S1.

$P G C-1 \alpha$ expression is correlated with the severity of cyanosis

Next, PGC- $1 \alpha$ mRNA expression was analyzed in the myocardial muscles of individual TOF patients. As shown in Figure 3, the PGC- $1 \alpha$ mRNA level in the heart tissue correlated inversely with the preoperative blood $\mathrm{O}_{2}$ saturation (Figure $3 \mathrm{~A}, r=-0.535, P=0.029$ ) but positively with the hematocrit of TOF patients (Figure 3B, $r=0.504, P=0.040$ ), indicating that PGC- $1 \alpha$ expression is positively correlated with the severity of cyanosis.

Table 1 Patient characteristics

\begin{tabular}{lccc}
\hline & Normal $(n=12)$ & TOF $(n=17)$ & $P$ \\
\hline $\mathrm{O}_{2}$ saturations (\%) & $98 \pm 2$ & $86 \pm 2$ & $<0.001$ \\
Hematocrit (\%) & $35 \pm 1$ & $51 \pm 2$ & $<0.001$ \\
$\mathrm{Hb}(\mathrm{g} / \mathrm{l})$ & $118 \pm 5$ & $170 \pm 7$ & $<0.001$ \\
\hline
\end{tabular}


A

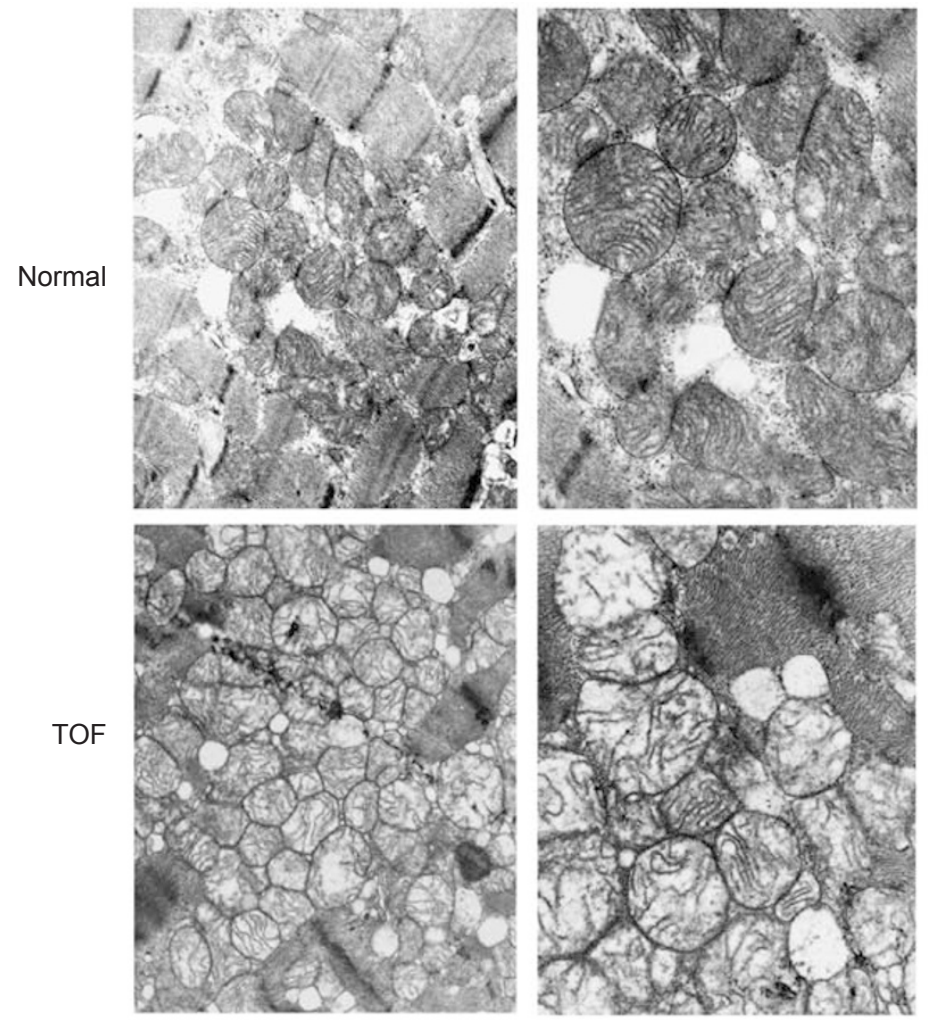

B

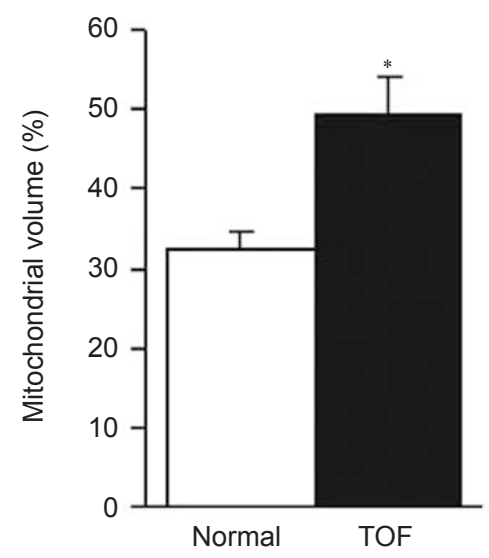

C

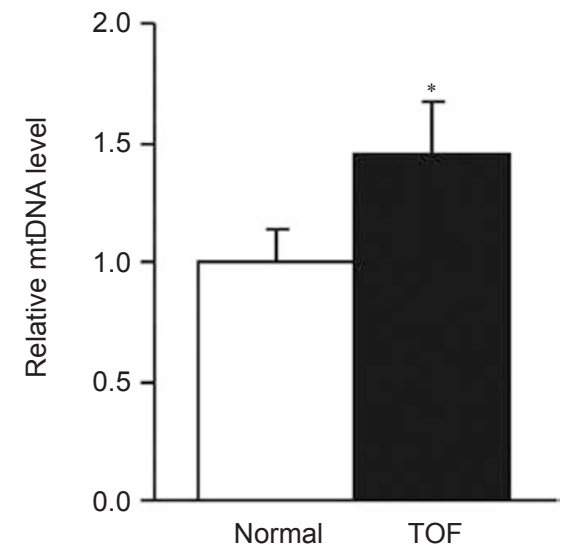

Figure 1 Electron microscopy images of longitudinal anterior sections from the myocardial muscle of control (top panels) and TOF (lower panels) subjects. Randomly selected areas were photographed at $10000 \times$ (left panels) or $20000 \times$ (right panels) magnification (A). Morphometric quantification of mitochondrial volume from 12 control and 17 TOF electron micrographs is shown (B). The relative mitochondrial copy number was determined by real-time PCR (C). $* P<0.05$ compared with control.

These results suggest that hypoxia may be a main cause of the elevated PGC- $1 \alpha$ expression in the myocardial muscles of TOF patients.

Hypoxia induces $P G C-1 \alpha$ expression in the $H 9 c 2$ cell line and in cultured primary cardiac myocytes

To further study the mechanism underlying the el- evated PGC-1 $\alpha$ expression in the myocardium of TOF patients, we determined the effect of hypoxia on PGC$1 \alpha$ expression in cultured $\mathrm{H} 9 \mathrm{c} 2$ cells and primary cardiac myocytes. As shown in Figure 4A, the PGC- $1 \alpha$ mRNA level in $\mathrm{H} 9 \mathrm{c} 2$ cells cultured under hypoxic conditions was increased more than 3-fold compared with that of the control group. The increase in mRNA levels of PGC-1 $\alpha$ 
A

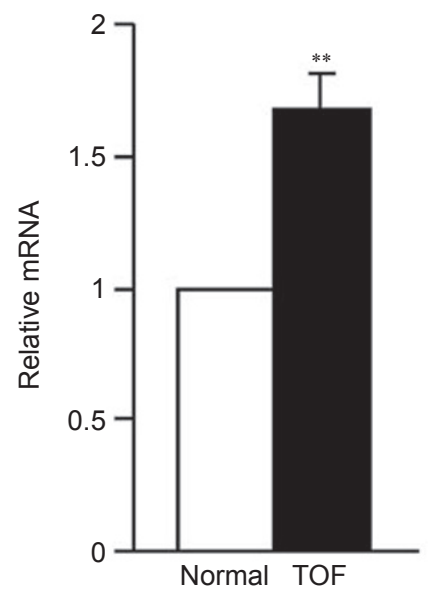

B
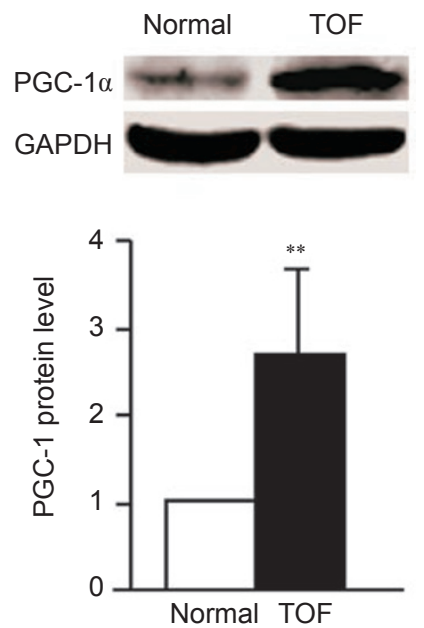

Figure 2 PGC-1 $\alpha$ expressions were elevated in the heart tissue of TOF patients compared with control subjects. PGC-1 $\alpha$ mRNA expression was determined by real-time PCR (A). Samples were normalized using the geometric mean of four housekeeping genes (HPRT1, RPLP0, POLR2A and $\beta$-actin). PGC-1 $\alpha$ protein levels were determined by western blotting with antiPGC-1 $\alpha$ antibody and by comparing to the blotting with anti-GAPDH antibody, and densitometry analysis of PGC-1 $\alpha$ is shown (B). Representative blots of three similar results are shown. The data represent 17 TOF and 12 control subjects. ${ }^{* *} P<0.01$ compared with control.

A

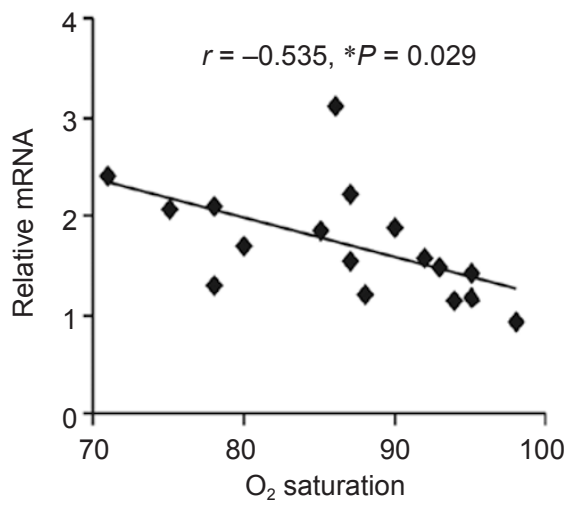

B

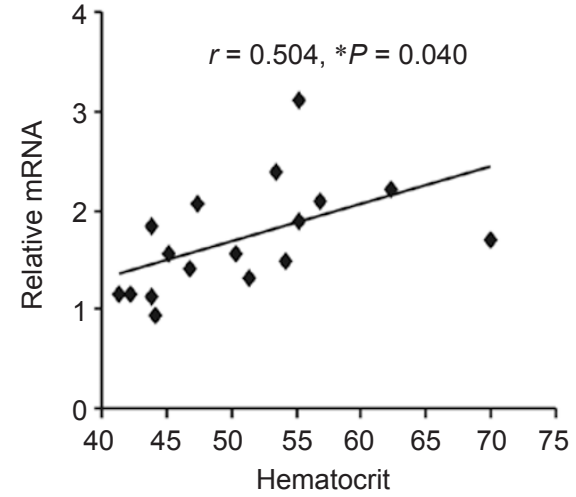

Figure 3 Positive correlation of PGC-1 $\alpha$ expression with severity of cyanosis. Lower $\mathrm{O}_{2}$ saturation (A) and higher hematocrit (B) were associated with higher expression of PGC- $1 \alpha$ mRNA. $* P<0.05$ compared with control.

was accompanied by a significant increase in the PGC- $1 \alpha$ protein level (Figure 4B). This trend was mirrored in the primary cardiac myocyte cultures. Under hypoxic conditions, the PGC-1 $\alpha$ mRNA expression increased by 1.6fold (Figure 4C) and the protein level increased by 2 -fold (Figure 4D). These results indicate that hypoxia increases PGC-1 $\alpha$ expression in cardiac myocytes and likely contributes to the elevation of PGC- $1 \alpha$ in the myocardium of TOF patients.

The AMPK signaling pathway is involved in hypoxiainduced PGC-1 $\alpha$ expression

AMPK is a heterotrimer composed of a catalytic $\alpha$ subunit and regulatory $\beta$ and $\gamma$ subunits. It is activated by the binding of AMP to two sites on the $\gamma$ subunit, leading to allosteric activation of AMPK and phosphorylation of the $\alpha$ subunit at Thr-172 $[27,28]$. It is generally accepted that AMPK functions to maintain the ATP supply in all eukaryotic cells. To determine whether the AMPK signaling pathway is involved in hypoxia-induced PGC$1 \alpha$ expression, we checked the phosphorylation levels of AMPK in primary cardiac myocytes cultured under normal and hypoxic conditions. The results showed that hypoxia markedly activated AMPK (Figure 5B), and this was associated with a 1.8-fold increase in the PGC$1 \alpha$ mRNA level and an approximately 1.5 -fold increase 
A

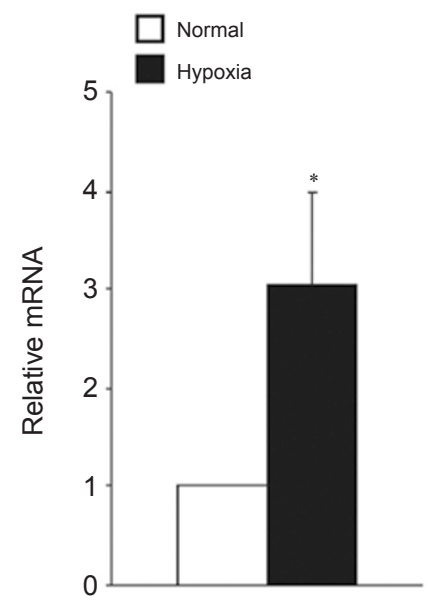

C

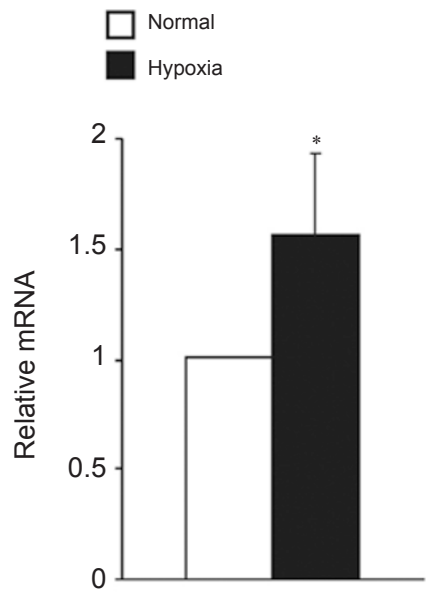

B
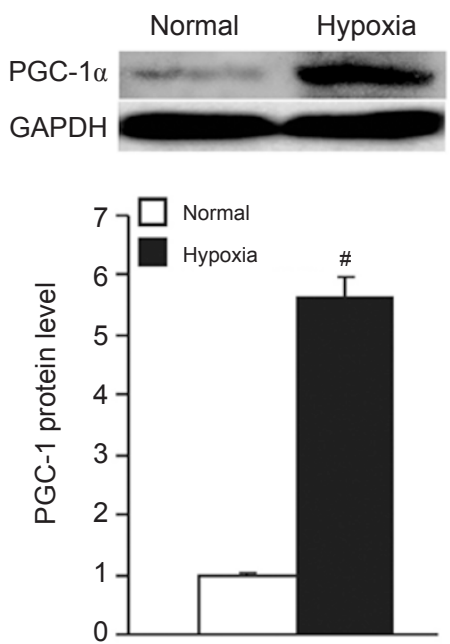

D
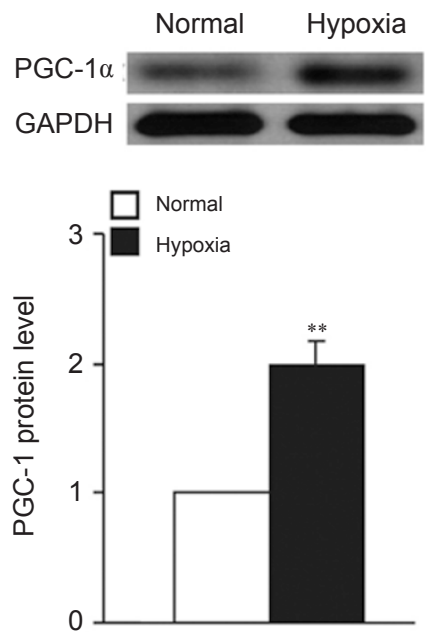

Figure 4 Hypoxia induces PGC-1 $\alpha$ expression in cultured cardiac myocytes. H9c2 cells (A and B) and primary cardiac myocytes (C, D) were cultured under normal or hypoxic conditions, and PGC-1 $\alpha$ expression was determined by real-time PCR (A, C) and by western blot with anti-PGC-1 $\alpha$ antibody compared with anti-GAPDH antibody (B, D). For western blots, the densitometry analysis of PGC- $1 \alpha$ is shown. The bars represent means $\pm \mathrm{SE}(n=6)$. ${ }^{*} P<0.05, * * P<0.01, \# P<0.001$ compared with control.

in the PGC-1 $\alpha$ protein level (Figure 5A and 5C). In the presence of $10 \mu \mathrm{M}$ AMPK inhibitor, the phosphorylation of AMPK was slightly blocked. However, when the concentration of compound $\mathrm{C}$ was increased to $40 \mu \mathrm{M}$, the activation of AMPK was largely abolished (Figure 5B). Interestingly, in the compound C-treated samples, the expression of both PGC- $1 \alpha$ mRNA and protein was decreased to a level that was even lower than that of normal conditions (Figure 5A and 5C). To further test the role of the AMPK pathway in hypoxia-induced PGC- $1 \alpha$ expression, we knocked down the expression of the $\alpha$ subunit of AMPK by siRNA interference. To achieve the highest efficiency of gene silencing, a combination of two siRNA oligonucleotide sequences derived from the two isoforms of the $\alpha$ subunit was introduced to the cells. As shown in Figure $5 \mathrm{E}$, the combined siRNAs significantly lowered aAMPK expression. Following AMPK knockdown, the stimulatory effect of hypoxia on PGC- $1 \alpha$ expression was abolished (Figure 5D and 5F). Together, these results indicate that the AMPK signaling pathway is involved in hypoxia-induced PGC- $1 \alpha$ expression.

Hypoxia induces the expression of key genes relating to mitochondrial biogenesis

There are several key genes, such as mitochondria DNA transcriptional factor A (TFAM), aminolevulinate 
A

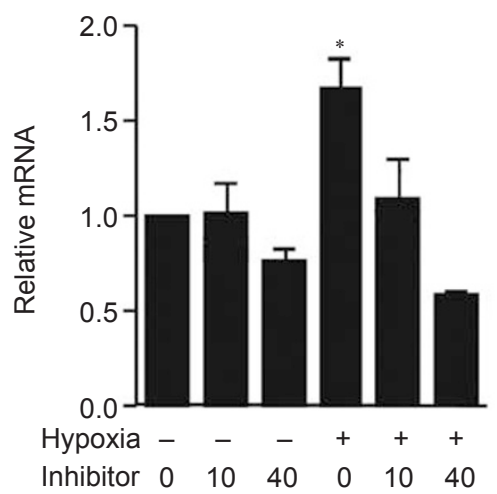

C

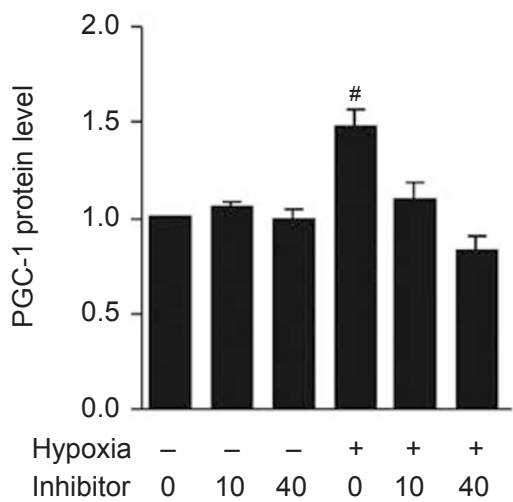

E

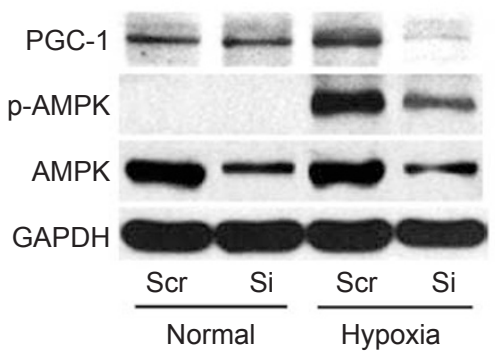

B

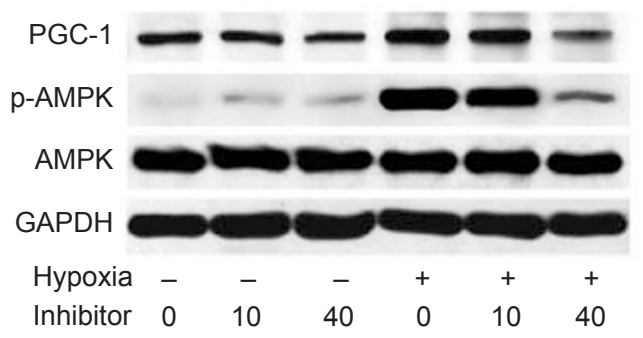

D

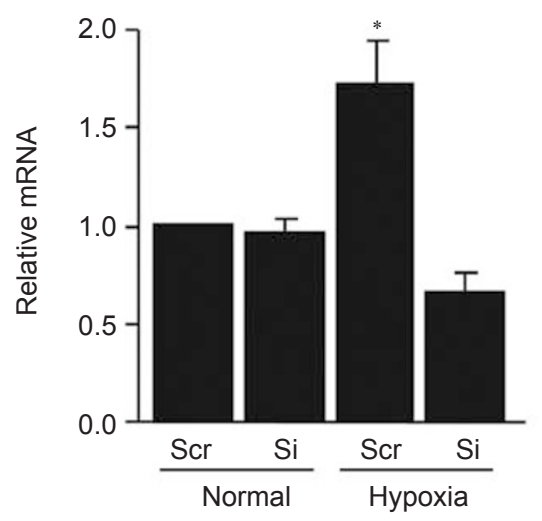

F

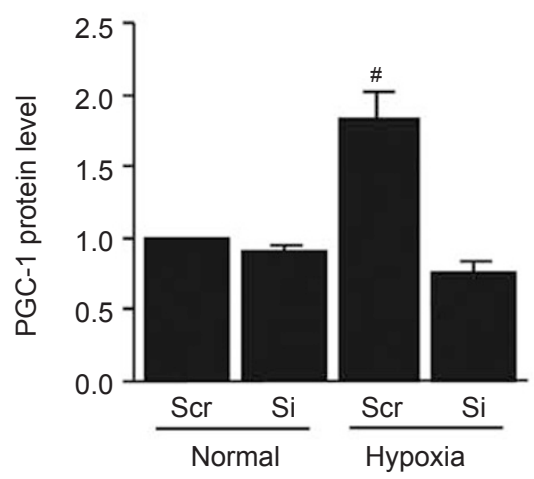

Figure 5 Hypoxia-induced PGC-1 $\alpha$ expression is mediated through the AMPK signaling pathway. Primary cardiac myocytes were cultured under normal or hypoxic conditions in the absence and presence of different doses of AMPK inhibitor (10 or 40 $\mu \mathrm{M})$. H9c2 cells transfected with siRNA ( $\mathrm{Si}$ ) or scrambled sequence ( $\mathrm{Src}$ ) were then cultured under normal or hypoxic conditions. PGC-1 $\alpha$ expression levels were determined by real-time PCR (A, D) and by western blot (B, E). AMPK activity was determined by western blotting with phos-AMPK antibody and by comparing to the blotting with total AMPK antibody. Densitometry analysis of PGC- $1 \alpha(\mathbf{C}, \mathbf{F})$ is shown. $* P<0.05$, \#P<0.001 compared with the control group.

synthase (ALAS) and cytochrome $c$ (Cyto $c$ ), that play critical roles in mitochondrial biogenesis and function $[19,29]$. To test whether the elevation of PGC- $1 \alpha$ expression by hypoxia is associated with up-regulation of the expression of these genes, we examined their mRNA levels in primary cardiac myocytes cultured under hypoxic conditions. As shown in Figure 6A, we found that the mRNA level of TFAM in the cells cultured under hypoxic conditions was increased 5-fold compared with that of the cells cultured under normal conditions after 12 
A

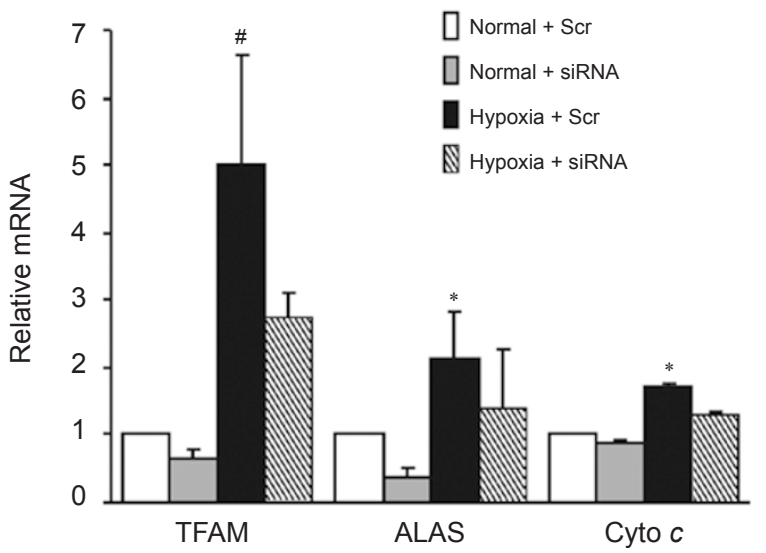

C

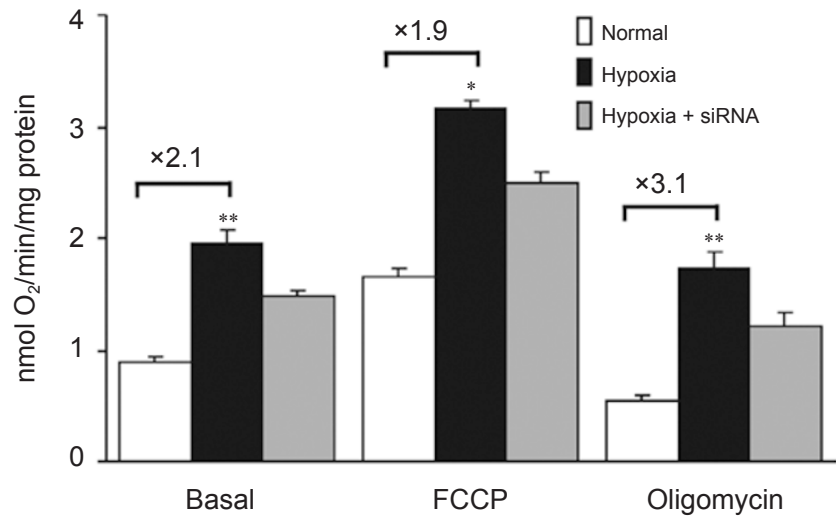

B

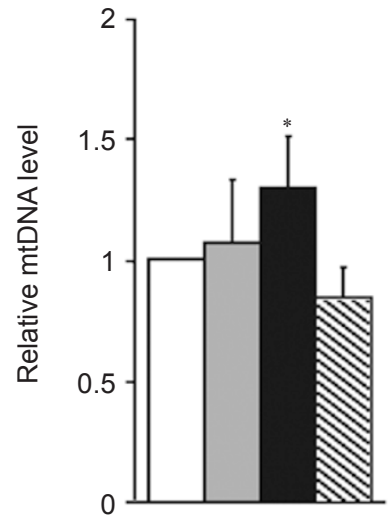

Figure 6 Hypoxia induces mitochondrial biogenesis in vitro. Cardiac myocytes transfected with scrambled sequence (Src) or PGC-1 $\alpha$ siRNA (siRNA) were cultured under normal or hypoxic conditions. TFAM, ALAS and cytochrome $c$ (Cyto $c$ ) mRNA levels were determined by real-time PCR (A). Relative mtDNA levels were determined by real-time PCR (B). Basal, maximum, and uncoupled respiration of these cells was determined (C). Each measurement was performed in triplicate and normalized to the protein content of the cells. $* P<0.05$, $* * P<0.01$, \#P<0.001 compared with the normal group.

h. Under hypoxic conditions, the mRNA levels of ALAS and Cyto $c$ in cardiac myocytes were increased 2.1-fold and 1.6-fold, respectively, compared with those under normal conditions (Figure 6A). Moreover, the mtDNA copy number was 1.3 -fold higher in the cells cultured under hypoxic conditions (Figure 6B).

To further investigate whether elevated expression of PGC- $1 \alpha$ increases the functional capacity for mitochondrial respiration in cardiac myocytes cultured under hypoxic conditions, oxygen consumption rates were determined. After $12 \mathrm{~h}$ of incubation in hypoxic conditions, the basal oxygen consumption of cardiac myocytes was about 2.1-fold higher than that of cells cultured under normal conditions (Figure 6C). The FCCP-stimulated oxygen consumption reflects the number and electron transport activity of mitochondria. Figure $6 \mathrm{C}$ shows that the FCCP-stimulated oxygen consumption was increased 1.9-fold in the hypoxia group. Oligomycin allows respiration through proton leak only. In the presence of this drug, the oxygen consumption was 3.1-fold higher in the hypoxia group. These results, together with the increased mitochondrial number observed in the myocardial muscle of TOF patients (Figure 1), suggest that hypoxia activates the mitochondrial biogenesis program in cardiac myocytes.

To test whether PGC- $1 \alpha$ is involved in hypoxia-stimulated mitochondrial gene expression and mtDNA replication, we used siRNA to knock down PGC-1 $\alpha$ expression. The efficiency of the siRNA we used was described previously $[30,31]$ and is also shown in Supplementary information, Figure S1. Following PGC-1 $\alpha$ knockdown, hypoxia-induced TFAM, ALAS and Cyto $c$ expression were partially abolished (Figure 6A). Meanwhile, the mtDNA levels returned to levels similar to those of cells 
cultured under normal conditions (Figure 6B). As shown in Figure 6C, the increased basal, maximal and uncoupled mitochondrial respiration levels observed in hypoxic conditions also partially reverted. These results indicate that the increased PGC-1 $\alpha$ expression is involved in, but not required for, the observed stimulation of mitochondrial biogenesis by hypoxia.

\section{Discussion}

Recently, it has been established that PGC-1 $\alpha$ expression is associated with tissue $\mathrm{O}_{2}$ content. PGC- $1 \alpha$ expression was reported to be induced by a lack of nutrients and oxygen and to contribute to the up-regulation of VEGF expression and angiogenesis in skeletal muscle [32]. Hypoxia was also found to increase PGC-1 $\alpha$ expression and stimulate mitochondrial biogenesis in the brain subcortex [33]. However, in a rat model of neonatal hypoxic/ischemic brain injury, PGC- $1 \alpha$ expression was found to be unchanged [34]. In our study, we found that in the myocardial muscle of patients with TOF, PGC- $1 \alpha$ expression is up-regulated (Figure 2). Sustained pressure overload in the right ventricle of the heart and chronic systemic hypoxia are the primary indications of TOF $[3,4]$. In previous studies, PGC- $1 \alpha$ expression was reported to be reduced in the heart tissue in several wellestablished pressure overload models $[21,25,26]$. We hypothesized that it is the lack of oxygen that causes the increase in PGC- $1 \alpha$ expression in the myocardium of TOF patients. Our results show that PGC- $1 \alpha$ expression in the myocardium of TOF patients is correlated with the severity of cyanosis (Figure 3) and that hypoxia induces PGC-1 $\alpha$ expression in cultured cardiac myocytes (Figure 4). We therefore conclude that hypoxia contributes at least partially to the elevation of PGC- $1 \alpha$ in the myocardium of TOF patients. This is the first report establishing the relationship between $\mathrm{O}_{2}$ content and PGC- $1 \alpha$ expression in cardiac myocytes.

The mechanism underlying hypoxia-induced PGC$1 \alpha$ expression was not clear. It has been shown that a hypoxic environment can induce PGC- $1 \alpha$ expression in a HIF-independent pathway [32]. The AMPK signaling pathway, a stress response pathway, has been reported to be involved in many adaptive processes that increase cell survival under a variety of conditions. It has been shown that hypoxia can activate the AMPK pathway in the myocardium $[35,36]$. In our study, we found that hypoxiainduced PGC- $1 \alpha$ expression was associated with the activation of AMPK. We also found that blocking the AMPK pathway, either by the addition of an AMPK inhibitor or by transfection of AMPK siRNAs, returned both PGC- $1 \alpha$ mRNA and protein expression levels to those of normally cultured cells (Figure 5), indicating that hypoxic conditions alter PGC- $1 \alpha$ expression through an AMPK-dependent pathway. We noted that the basal level of PGC$1 \alpha$ seemed to be affected by manipulation of the AMPK pathway (Figure 5A, 5C, 5D and 5F), suggesting that the AMPK pathway is also important for maintaining the basal level of PGC- $1 \alpha$ in cardiac myocytes. AICAR, an AMP-activated protein kinase agonist, has been shown to increase PGC- $1 \alpha$ expression and increase mitochondrial biogenesis and activity in skeletal muscle tissue [37, 38]. Our results further describe the positive effect of AMPK activation on PGC- $1 \alpha$ expression and mitochondrial biogenesis and also suggest a new strategy to activate the AMPK pathway and thus increase PGC- $1 \alpha$ expression.

One of the primary functions of PGC- $1 \alpha$ is the activation of mitochondrial biogenesis and oxidative phosphorylation [16-19]. Over-expression of PGC-1 $\alpha$ can increase mitochondrial number and activity in cultured cardiac myocytes [24] and in the heart tissue of mice [39]. Hearts from PGC-1 $\alpha$ knockout mice show reduced mitochondrial enzymatic activities and decreased levels of ATP [21]. These gain- or loss-of-function experiments have demonstrated that PGC- $1 \alpha$ is able to activate the full programs of mitochondrial biogenesis and oxidative phosphorylation in the cardiac muscle bed. In the present study, PGC-1 $\alpha$ expression was found to be significantly higher in the myocardium from TOF patients compared with control subjects. Given the fundamental role of PGC- $1 \alpha$ in mitochondrial biogenesis in the cardiac muscle, we investigated the mitochondria in the myocardium using transmission electron microscopy. We found mitochondrial number to be increased in TOF patients. Meanwhile, the relative mitochondrial DNA copy number was statistically significantly higher in the myocardium of TOF patients (Figure 1). TFAM is a nuclear encoded factor that translocates to the mitochondria, where it binds to the D loop of mitochondrial DNA and activates its replication and transcription. TFAM has been shown to be regulated by PGC- $1 \alpha$ through coactivation with NRFs [19]. Elevation of PGC-1 $\alpha$ expression has also been reported to increase the expression of genes critical for mitochondrial function, such as ALAS and Cyto $c$. In our experiments, the expression levels of these genes were markedly increased under hypoxic conditions in cultured primary cardiac myocytes (Figure 6). Furthermore, the basal, uncoupled and maximum respiration rates were significantly higher in the cells cultured under hypoxic conditions compared with those of the normal group. When PGC- $1 \alpha$ expression was knocked down by siRNA, the increase in expression of these genes was partially abolished, suggesting that hypoxia-induced PGC-1 $\alpha$ expression was involved in the up-regulation of mitochon- 
drial gene expression. However, our data also indicate that although PGC- $1 \alpha$ has a critical role in mitochondrial biogenesis, it is not absolutely required for mitochondrial biogenesis. This is consistent with a previous observation that PGC-1 $\alpha$ knockout mice have normal tissue structure and mitochondrial biogenesis in the myocardium [21]. Additional regulators of mitochondrial biogenesis were investigated by microarray analysis, and expression of many of these genes was found to be slightly increased (Supplementary information, Figure S2).

The increase in PGC-1 $\alpha$ expression and mitochondrial biogenesis in the myocardium of TOF patients could potentially be a compensatory mechanism to meet the demands for ATP in an attempt to minimize hypoxic damage. The survival of TOF patients is dependent on many adaptive mechanisms to improve $\mathrm{O}_{2}$ delivery and energy production along with mechanisms that minimize reoxygenation injury [5-7]. Under hypoxic conditions, because of the insufficient $\mathrm{O}_{2}$ supply, the production of ATP by each mitochondrion is decreased. To meet the energy supply, the cells must increase the number of the mitochondria to produce more ATP. Thus, the elevation of PGC-1 $\alpha$ expression and mitochondrial number found in our study could be a mechanism by which the cardiac myocytes attempt to increase the energy output. The data from microarray analysis showed that under hypoxic conditions, the glycolytic pathway was markedly activated in cultured cardiac myocytes (Supplementary information, Figure S3), suggesting a switch of fuel selection. However, in the myocardium of TOF patients, activation of the glycolytic pathway is less obvious, indicating that the in vivo conditions are more complicated. Meanwhile, chronic hypoxia in vivo may cause other adaptive changes to restore the fuel selection. There has been a report that showed a switch of mitochondrial metabolic properties to better use oxygen under hypoxic conditions [40]. In our experiments, we also found that the Cox 4 isoform 1 (Cox4I1) expression is slightly lower in TOF hearts, whereas the Cox 4 isoform 2 (Cox4I2) expression is about 1.3-fold higher in TOF hearts compared with control. In the cells cultured under hypoxic conditions, Cox $4 \mathrm{I} 2$ expression was increased by 1.7 -fold compared with the control group; meanwhile, the Cox4I1 expression was not significantly altered (Supplementary information, Figure S2). All of these results indicate that several adaptive mechanisms exist in the cardiac myocytes. It also has been shown that hypoxia induces PGC$1 \alpha$ expression, VEGF expression and angiogenesis in muscle cells, aiding the body in improving $\mathrm{O}_{2}$ delivery [32]. Our new findings provide additional insights to address the adaptive mechanism by which the body attempts to minimize injuries due to hypoxic conditions.
However, prolonged challenges to the heart may cause abnormal mitochondrial activity and morphology. Several reports showed decreased mitochondrial enzyme activity in TOF hearts $[12,13]$. Our results also showed vacuolar degeneration and breakage of the mitochondrial cristae in the myocardium of TOF patients (Figure 1A). These results indicate that although cells try to meet the energy demand by increasing the mitochondrial number, early surgical repair of TOF is necessary to minimize organ damage, and the elevation of PGC- $1 \alpha$ expression could be helpful to patients who are suffering from systemic hypoxia.

In summary, our results indicate that hypoxic conditions increase the expression of PGC- $1 \alpha$ and mitochondrial biogenesis in cardiac myocytes. The up-regulation of PGC- $1 \alpha$ is mediated through an AMPK-dependent pathway. These effects could be an adaptive mechanism in an attempt to increase ATP output and minimize hypoxic damage to the heart. Thus, this study suggests that patients who are suffering from systemic hypoxia could potentially benefit from the elevation of PGC- $1 \alpha$ expression.

\section{Materials and Methods}

\section{Chemicals and reagents}

DMEM F12 medium and compound C (AMPK inhibitor) were purchased from Sigma. DMEM, antibiotics, trypsin and fetal calf serum were from Gibco-Invitrogen. The RNeasy mini kit and RNase-free DNase set were purchased from Qiagen (Hilden, Germany). All other reagents were of analytical grade.

\section{Study population}

The study population includes 17 patients with TOF who underwent primary complete repair at Nanjing Drum Tower Hospital from 2004 to 2006. The patients with TOF, 10 male and 7 female, were between 1.5 and 36 years old. The control group consisted of 12 normal subjects who were deceased from non-cardiac causes (Supplementary information, Table S1). Informed consent was obtained from each individual before the surgery, and the study protocol was approved by the Institutional Review Board of Nanjing University, Nanjing, China. This investigation was carried out in accordance with the principles outlined in the Declaration of Helsinki.

\section{Sampling}

In all patients undergoing primary repair, muscle bundles that are routinely resected from the right ventricular outflow tract as part of the surgery were recovered. Myocardium specimens were either immersed in liquid nitrogen and transferred to a $-80{ }^{\circ} \mathrm{C}$ freezer or were cut into $1-2 \mathrm{~mm}^{3}$ size pieces, immediately fixed for $12 \mathrm{~h}$ in cold $3 \%$ glutaraldehyde in sodium cacodylate buffer, and set aside for ultra-structural evaluation.

\section{Electron microscopic study}

Samples were fixed in osmium tetroxide buffered to $\mathrm{pH} 7.4$ 
with phosphate. The tissues were then dehydrated in ethanol and propylene oxide and embedded in Maraglas epoxy resin, sectioned, and examined with a transmission electron microscope as previously described $[41,42]$. Twenty randomly selected areas were photographed at $10000 \times$ or $20000 \times$ magnification. The mitochondrial volume densities were determined in a blinded fashion using the principles of Weibel and Steer as previously described [24].

\section{Cell culture}

$\mathrm{H} 9 \mathrm{c} 2$ cells derived from embryonic rat ventricle (purchased from Cell Bank of Shanghai Institute of Biochemistry and Cell Biology, China) were cultured in DMEM supplemented with $10 \%$ fetal bovine serum, $100 \mathrm{U} / \mathrm{ml}$ penicillin and $100 \mu \mathrm{g} / \mathrm{ml}$ streptomycin in an atmosphere of $95 \%$ air, $5 \% \mathrm{CO}_{2}$ at $37{ }^{\circ} \mathrm{C}$. Primary rat neonatal cardiac myocytes were prepared from 1-day-old Harlan Sprague-Dawley rats as described $[43,44]$ and were cultured in DMEM F12 medium. Primary cardiac myocytes and H9c2 cells were subjected to normoxic or hypoxic conditions at $70 \%-80 \%$ confluence for $24 \mathrm{~h}$. The hypoxic environment was created by the infusion of a pre-analyzed gas mixture of $95 \% \mathrm{~N}_{2}$ and $5 \% \mathrm{CO}_{2}$ into an airtight modular chamber (Billups-Rothenberg, Inc.) as described previously [45, 46]. Compound C (Sigma, P5499), an AMPK inhibitor, was dissolved in DMSO and then further diluted in cell culture medium to different concentrations. All animals were treated in accordance with institutional animal-handling guidelines. This investigation was carried out in accordance with the Guide for the Care and Use of Laboratory Animals published by the US National Institutes of Health (NIH Publication No. 8523, revised 1996).

\section{siRNA interference}

All small interfering RNAs were synthesized by Invitrogen Biotechnology. The 19-nucleotide (plus two nucleotides at the 5' end) siRNA sequences were as follows: $\alpha 1$-AMPK, GCA UAU GCU GCA GGU AGA U; $\alpha 2-A M P K$, CGU CAU UGA UGA UGA GGC U; PGC-1 $\alpha$, GAC GGA TTG CCC TCA TTT G; and scrambled siRNA, AUU GUA UGC GAU CGC AGA C. The combination of $\alpha 1-$ and $\alpha 2$-AMPK siRNA was transfected into cells to achieve the most effective gene silencing as described previously [47, 48]. All siRNAs were transfected into cells using Lipofectamine reagent (Gibco-Invitrogen, Carlsbad, CA, USA) according to the manufacturer's manual.

\section{mtDNA copy number assay}

The relative mtDNA copy number was determined by real-time quantitative PCR as described previously [49, 50]. Genomic DNA was extracted from human samples or cardiac myocytes using the QIAamp DNA isolation kit (Qiagen, Hilden, Germany). Mitochondrial DNA (mtDNA) and nuclear DNA (nDNA) copy number was determined by real-time PCR using SYBR Green on an ABI 7300 Thermocycler. Human mtDNA was amplified between nucleotide positions 3459 and 3569, while rat mtDNA was amplified between nucleotide positions 2469 and 2542. The human globulin gene and rat GAPDH gene were selected for the quantification of nDNA copies. All samples were run in triplicate. Relative copy number was calculated from the threshold cycle value ( $\Delta C \mathrm{t}$ value). All primers used are listed in Supplementary information, Table S2.

\section{Cellular respiration assay}

Whole-cell respiration was measured as described before [19].
Briefly, cells were washed with PBS and detached by trypsinization. They were then resuspended in PBS and transferred to a 2-ml Clark-type oxygen electrode chamber that was connected to a circulating water bath at $37^{\circ} \mathrm{C}$. Basal respiration was measured, and then nonphosphorylating (uncoupled) respiration was determined in the presence of the ATP synthase inhibitor oligomycin $(2.5 \mu \mathrm{g} /$ $\mathrm{ml})$. The maximum respiration of cells, induced with $2 \mu \mathrm{M}$ FCCP, was measured. After finishing the measurements, cells were collected from the chamber, and their protein concentrations were determined using the BCA kit (Pierce).

\section{RNA isolation and analysis}

Total RNA was isolated from human samples, primary cardiac myocytes and H9c2 cells using the RNeasy kit according to the manufacturer's instructions. cDNA was synthesized and subjected to PCR amplification using the ABI 7300 Thermocycler. The relative abundance of mRNA was determined from the $\mathrm{Ct}$ values and was normalized to the geometric mean value of several housekeeping genes. RPLP0, HPRT1, POLR2A and $\beta$-actin were used as human housekeeping genes, while HPRT1, SDHA and $\beta$-actin were used as rat housekeeping genes. All primers used are listed in Supplementary information, Table S2.

\section{Western blot analysis}

For western blot analysis, $60 \mu \mathrm{g}$ of the total protein extracted from heart tissue and harvested cells was used in 7.5\% SDSPAGE gel electrophoresis for PGC-1 $\alpha$ detection. Rabbit polyclonal PGC- $1 \alpha$ antibody (Santa Cruz Biotechnology, USA) and mouse monoclonal GAPDH antibody (Santa Cruz) were used as primary antibodies. Expression level was quantified by densitometry with NIH Image software using three independent western blots.

\section{Statistical analysis}

All results are expressed as means \pm SEM. Data were analyzed using one-way ANOVA and Student-Newman-Keuls tests for multiple comparisons or Student's $t$ tests for unpaired data. In all cases, $P \leq 0.05$ was considered as statistically significant.

\section{Acknowledgments}

We thank Yun Luo, Jia Jia and Dr Yun Xu at Nanjing University, Nanjing, China, for their scientific discussions and experimental assistance. This work was supported by grants from the National Natural Science Foundation of China (Nos. 30871195, 90813035, 30890044), the National Basic Research Program of China (Nos. 2006CB503909, 2004CB518603), the "111" Project, the Hi-Tech Research and Development Program of China (No. 2006AA02A112,), and the Natural Science Foundation of Jiangsu Province (Nos. BK2004082, BK2006714, BK2008021).

\section{References}

1 Moran AM, Hornberger LK, Jonas RA, Keane JF. Development of a double-chambered right ventricle after repair of tetralogy of Fallot. J Am Coll Cardiol 1998; 31:1127-1133.

2 Sommer RJ, Hijazi ZM, Rhodes JF. Pathophysiology of congenital heart disease in the adult: part III: Complex congenital heart disease. Circulation 2008; 117:1340-1350.

3 Tongsong T, Sittiwangkul R, Chanprapaph P, Sirichotiyakul 
S. Prenatal sonographic diagnosis of tetralogy of fallot. J Clin Ultrasound 2005; 33:427-431.

4 Yang X, Freeman LJ, Ross C. Unoperated tetralogy of Fallot: case report of a natural survivor who died in his 73rd year; is it ever too late to operate? Postgrad Med J 2005; 81:133-134.

5 Reddy S, Osorio JC, Duque AM, et al. Failure of right ventricular adaptation in children with tetralogy of Fallot. Circulation 2006; 114:I37-I42.

6 Chi NC, Karliner JS. Molecular determinants of responses to myocardial ischemia/reperfusion injury: focus on hypoxia-inducible and heat shock factors. Cardiovasc Res 2004; 61:437447.

7 Chen WJ, Chen HW, Yu SL, et al. Gene expression profiles in hypoxic preconditioning using cDNA microarray analysis: altered expression of an angiogenic factor, carcinoembryonic antigen-related cell adhesion molecule 1. Shock 2005; 24:124131.

8 Isomura T, Hisatomi $\mathrm{K}$, Inuzuka $\mathrm{H}$, et al. Ultrastructural alterations of right and left ventricular myocytes in tetralogy of Fallot. Kurume Med J 1990; 37:177-183.

9 Moreno AJ, Santos DJ, Palmeira CM. Ischemic heart disease: the role of mitochondria - carvedilol prevents lipid peroxidation of mitochondrial membranes. Rev Port Cardiol 1998; 17 Suppl 2:II63-II77.

10 DiMauro S, Hirano M. Mitochondria and heart disease. Curr Opin Cardiol 1998; 13:190-197.

11 Ferrari R. The role of mitochondria in ischemic heart disease. J Cardiovasc Pharmacol 1996; 28 Suppl 1:S1-S10.

12 Maurer I, Zierz S. Mitochondrial respiratory chain enzyme activities in tetralogy of Fallot. Clin Investig 1994; 72:358363.

13 Shinde SB, Save VC, Patil ND, Mishra KP, Tendolkar AG. Impairment of mitochondrial respiratory chain enzyme activities in tetralogy of Fallot. Clin Chim Acta 2007; 377:138-143.

14 Kniazeva GD, Sheremet'eva GF, Konstantinov BA, Kostriukov BM, Liubarskaia AD. (Intraoperative damage to the myocardium and heart failure upon radical correction of the tetralogy of Fallot). Arkh Patol 1979; 41:23-29.

15 Toussaint M, Planche C, Duboc D, et al. Left ventricular ultrastructure in pulmonary stenosis and in tetralogy of Fallot. Virchows Arch A Pathol Anat Histopathol 1987; 411:33-38.

16 Puigserver P, Wu Z, Park CW, et al. A cold-inducible coactivator of nuclear receptors linked to adaptive thermogenesis. Cell 1998; 92:829-839.

17 Puigserver P, Spiegelman BM. Peroxisome proliferatoractivated receptor-gamma coactivator 1 alpha (PGC-1 alpha): transcriptional coactivator and metabolic regulator. Endocr Rev 2003; 24:78-90.

18 Lin J, Handschin C, Spiegelman BM. Metabolic control through the PGC-1 family of transcription coactivators. Cell Metab 2005; 1:361-370.

19 Wu Z, Puigserver P, Andersson U, et al. Mechanisms controlling mitochondrial biogenesis and respiration through the thermogenic coactivator PGC-1. Cell 1999; 98:115-124.

20 Finck BN, Kelly DP. Peroxisome proliferator-activated receptor gamma coactivator-1 (PGC-1) regulatory cascade in cardiac physiology and disease. Circulation 2007; 115:25402548.

21 Arany Z, He H, Lin J, et al. Transcriptional coactivator PGC-
1 alpha controls the energy state and contractile function of cardiac muscle. Cell Metab 2005; 1:259-271.

22 Huss JM, Kelly DP. Mitochondrial energy metabolism in heart failure: a question of balance. J Clin Invest 2005; 115:547555.

23 Sano M, Abdellatif M, Oh H, et al. Activation and function of cyclin T-Cdk9 (positive transcription elongation factor-b) in cardiac muscle-cell hypertrophy. Nat Med 2002; 8:1310-1317.

24 Lehman JJ, Barger PM, Kovacs A, et al. Peroxisome proliferator-activated receptor gamma coactivator-1 promotes cardiac mitochondrial biogenesis. J Clin Invest 2000; 106:847-856.

25 Witt H, Schubert C, Jaekel J, et al. Sex-specific pathways in early cardiac response to pressure overload in mice. $J \mathrm{Mol}$ Med 2008; 86:1013-1024.

26 Lehman JJ, Kelly DP. Transcriptional activation of energy metabolic switches in the developing and hypertrophied heart. Clin Exp Pharmacol Physiol 2002; 29:339-345.

27 Hawley SA, Selbert MA, Goldstein EG, et al. 5'-AMP activates the AMP-activated protein kinase cascade, and $\mathrm{Ca}^{2+}$ / calmodulin activates the calmodulin-dependent protein kinase I cascade, via three independent mechanisms. J Biol Chem 1995; 270:27186-27191.

28 Arad M, Seidman CE, Seidman JG. AMP-activated protein kinase in the heart: role during health and disease. Circ Res 2007; 100:474-488.

29 Lin J, Wu H, Tarr PT, et al. Transcriptional co-activator PGC1 alpha drives the formation of slow-twitch muscle fibres. Nature 2002; 418:797-801.

30 Zhu L, Sun G, Zhang H, et al. PGC-1alpha is a key regulator of glucose-induced proliferation and migration in vascular smooth muscle cells. PLoS One 2009; 4:e4182.

31 Zhang Y, Liu C, Zhu L, et al. PGC-1alpha inhibits oleic acid induced proliferation and migration of rat vascular smooth muscle cells. PLoS One 2007; 2:e1137.

32 Arany Z, Foo SY, Ma Y, et al. HIF-independent regulation of VEGF and angiogenesis by the transcriptional coactivator PGC-1alpha. Nature 2008; 451:1008-1012.

33 Gutsaeva DR, Carraway MS, Suliman HB, et al. Transient hypoxia stimulates mitochondrial biogenesis in brain subcortex by a neuronal nitric oxide synthase-dependent mechanism. J Neurosci 2008; 28:2015-2024.

34 Yin W, Signore AP, Iwai M, et al. Rapidly increased neuronal mitochondrial biogenesis after hypoxic-ischemic brain injury. Stroke 2008; 39:3057-3063.

35 Horman S, Beauloye C, Vertommen D, et al. Myocardial ischemia and increased heart work modulate the phosphorylation state of eukaryotic elongation factor-2. J Biol Chem 2003; 278:41970-41976.

36 Yang J, Holman GD. Insulin and contraction stimulate exocytosis, but increased AMP-activated protein kinase activity resulting from oxidative metabolism stress slows endocytosis of GLUT4 in cardiomyocytes. J Biol Chem 2005; 280:40704078.

37 Jorgensen SB, Wojtaszewski JF, Viollet B, et al. Effects of alpha-AMPK knockout on exercise-induced gene activation in mouse skeletal muscle. FASEB J 2005; 19:1146-1148.

38 Lee WJ, Kim M, Park HS, et al. AMPK activation increases fatty acid oxidation in skeletal muscle by activating PPARalpha and PGC-1. Biochem Biophys Res Commun 2006; 
340:291-295.

39 Russell LK, Mansfield CM, Lehman JJ, et al. Cardiac-specific induction of the transcriptional coactivator peroxisome proliferator-activated receptor gamma coactivator- $1 \alpha$ promotes mitochondrial biogenesis and reversible cardiomyopathy in a developmental stage-dependent manner. Circ Res 2004; 94:525-533.

40 Fukuda R, Zhang H, Kim JW, et al. HIF-1 regulates cytochrome oxidase subunits to optimize efficiency of respiration in hypoxic cells. Cell 2007; 129:111-122.

41 Zhang Y, Ba Y, Liu C, et al. PGC-1alpha induces apoptosis in human epithelial ovarian cancer cells through a PPARgammadependent pathway. Cell Res 2007; 17:363-373.

42 Mannella CA, Marko M, Penczek P, Barnard D, Frank J. The internal compartmentation of rat-liver mitochondria: tomographic study using the high-voltage transmission electron microscope. Microsc Res Tech 1994; 27:278-283.

43 Burton PB, Raff MC, Kerr P, Yacoub MH, Barton PJ. An intrinsic timer that controls cell-cycle withdrawal in cultured cardiac myocytes. Dev Biol 1999; 216:659-670.

44 Disch DL, Rader TA, Cresci S, et al. Transcriptional control of a nuclear gene encoding a mitochondrial fatty acid oxidation enzyme in transgenic mice: role for nuclear receptors in cardiac and brown adipose expression. Mol Cell Biol 1996;
16:4043-4051.

45 Huss JM, Levy FH, Kelly DP. Hypoxia inhibits the peroxisome proliferator-activated receptor alpha/retinoid X receptor gene regulatory pathway in cardiac myocytes: a mechanism for $\mathrm{O}_{2}$-dependent modulation of mitochondrial fatty acid oxidation. J Biol Chem 2001; 276:27605-27612.

46 Wong HR, Menendez IY, Ryan MA, Denenberg AG, Wispe JR. Increased expression of heat shock protein-70 protects A549 cells against hyperoxia. Am J Physiol 1998; 275:L836L841.

47 Konrad D, Rudich A, Bilan PJ, et al. Troglitazone causes acute mitochondrial membrane depolarisation and an AMPKmediated increase in glucose phosphorylation in muscle cells. Diabetologia 2005; 48:954-966.

48 Li J, Yu X, Pan W, Unger RH. Gene expression profile of rat adipose tissue at the onset of high-fat-diet obesity. Am J Physiol Endocrinol Metab 2002; 282:E1334-E1341.

49 Lebrecht D, Kirschner J, Geist A, Haberstroh J, Walker UA. Respiratory chain deficiency precedes the disrupted calcium homeostasis in chronic doxorubicin cardiomyopathy. Cardiovasc Pathol 2009 Sep 9. doi:10.1016/j.carpath.2009.06.006.

50 Cree LM, Patel SK, Pyle A, et al. Age-related decline in mitochondrial DNA copy number in isolated human pancreatic islets. Diabetologia 2008; 51:1440-1443.

(Supplementary information is linked to the online version of the paper on the Cell Research website.) 